38.

\section{Incidence and Nature of Complaints Received in an Urban Fire Rescue System}

Todd J. LeDuc, BA, NREMT-P, * William E. Goellner, MD, FACEP, Nabil El Sanadi, MD, MBA, FACEP

Broward County Fire Rescue Division and Boward General Medical Center, ,Ft. Lauderdale, Florida USA

Objective: To determine the frequency and nature of patient complaints in a large urban Fire Rescue system.

Methods: A two year retrospective review of all unsolicited patient complaints received from any source either internal or external, through our full-time customer service personnel. All customer complaints, concerns and interactions are documented on an interaction form which we reviewed. All complaints were resolved with disposition related to the customer prior to our retrospective study inclusion.

Results: During the study period our Fire Rescue system responded to a total of 169,651 calls for medical assistance. Our division customer service personnel received a total of 87 unsolicited customer complaints $(n=87)$ regarding the service encounter with Fire Rescue/EMS. This accounts for a $0.0005 \%$ rate of customer complaints captured in correlation to total incidents during the study period. The majority of complaints, $27 \%$ were related to lost or missing goods. Service that was perceived as uncourteous or inconsiderate after calling 911 , accounted for $25 \%$ of complaints. Complaints regarding not being transported after calling 911 accounted for $18 \%$. Uncourteous driving or noise of sirens accounted for $7 \%$ of complaints. $7 \%$ of complaints resulted from not being transported to the hospital of their choice. Actual care received concerns were only $3 \%$ of total. Leaving medical objects behind was $2 \%$ of the total volume and $4 \%$ fit no specific trend.

Conclusion: In a large urban Fire Rescue system, we found our unsolicited complaint incidence low. Unsolicited complaints are not the most reliable source, however, they offer a valuable needs assessment and an opportunity for defining areas of improvement in customer service. We recommend customer skills training in all EMS services.
41.

Gastric Trauma and Pulmonary Aspiration at Autopsy After Cardiopulmonary Resuscitation William B. Felegi, DO ${ }^{1 *}$ Richard L. Doolittle, $P h D,{ }^{2}$

Alfred S. Conston, $M D,{ }^{3}$ Stephen V. Chandler, $M D^{3}$

1. Department of Emergency Medicine, Morristown Memorial Hospital, Morristown, New Jersey USA

2. Department of Biology, Rochester Institute of Technology, Rochester, New York USA

3. Department of Pathology, Somerset Medical Center, Somerville, New Jersey USA

Purpose: To determine the prevalence of gastric trauma and pulmonary aspiration after cardiopulmonary resuscitation (CPR) and to examine factors which may be associated with increased prevalence of these complications.

Methods: Retrospective review of 1,928 consecutive autopsy reports in a suburban teaching hospital from January 1, 1977 June 1, 1986 of which 860 received CPR and 1,037 did not. Of the 860 who received CPR, 527 received prehospital CPR and 333 arrested in hospital and received only hospital CPR. Thirtyone cases were excluded because of inability to accurately examine the stomach.

Results: There were 3 cases of stomach mucosal laceration and 4 cases of through and through laceration of the stomach in the CPR group and only one occurred in the non-CPR group $(p<0.03)$. All gastric trauma in the CPR group occurred in the prehospital group $(p<0.05)$. Pulmonary aspiration occurred in $31 \%$ of prehospital CPR patients and only $10 \%$ of hospital CPR patients $(p<0.0001)$. The prehospital group differed from the hospital group in the following factors: longer basic life support times without intubation, 14.0 minutes vs. 2.0 minutes $(p<0.0001)$ and longer total resuscitation time 46.9 minutes vs. 34.6 minutes $(p<0.0001)$.

Conclusion: Gastric trauma occurs infrequently. We found a prevalence of gastric trauma of $0.8 \%$ in prehospital patients and none in hospitalized patients following CPR. This is significantly lower than that reported in the literature of 9-12\%. Pulmonary aspiration occurs much more commonly than gastric trauma. There was a threefold increase of pulmonary aspiration in the prehospital CPR group as compared to the hospital CPR group. Factors which may be associated with increased gastric trauma and pulmonary aspiration were longer BLS and total resuscitation times. 